\title{
Addendum
}

\section{The Wieland-Miescher Ketone: A Journey from Organocatalysis to Natural Product Synthesis}

Ben Bradshaw, * Josep Bonjoch* Synlett 2011, 23, 337.

In the advance (e-first) version of this article reference 1 was missing twice (sections 2.1 and 2.3). The current online version has been corrected. 